\title{
Comment organiser le retour d'expérience
}

\author{
How to organise the post disaster report \\ par Ph. Huet \\ Ingénieur Général du Génie Rural, des Eaux et des Forêts \\ Mission d'inspection spécialisée de l'environnement
}

Avertissement : cet article a été écrit avant les catastrophes de l'Aude et des tempêtes de décembre 1999. Ces événements dramatiques rendent plus nécessaire encore la mise en œuvre rapide des mesures préconisées.

Since 1982, natural disasters, including floods, can be registered by the State within the framework of the compensation law for damage caused by naturel disasters. The last decade, which is also known as the IDNDR decade thanks to the UNO, has faced in our country numerous serious and sometimes fatal floods. This has led the authorities to investigate the methods of post disaster report. This paper takes stock of the meanings related to this field in the State Department in charge of Environmental Issues.

\section{1 - INTRODUCTION}

Selon l'ouvrage "Introduction aux Cyndiniques" [1] publié récemment sous la conduite du pôle cyndinique de l'Ecole des Mines de Paris, le retour d'expérience pour les risques est l'analyse détaillée d'un événement passé, dans le but d'améliorer les connaissances et de renforcer, si nécessaire, la chaîne de sécurité. Dans le domaine des risques naturels et donc de l'inondation, cette chaîne de sécurité comprend l'aménagement, c'est-à-dire la localisation des équipements et constructions, les dispositions constructives, les ouvrages de protection et la gestion des milieux, la surveillance et l'alerte, la gestion de la crise, et de la post-crise, le retour à la normale (procédure d'indemnisation "CATNAT" notamment, reconstruction, réaménagement). L'ensemble doit s'ancrer sur la recherche, la formation et l'information préventive (cf rapport Bourrelier).[2]

Pour les risques naturels, les "expériences de retour d'expérience" sont, à ce jour, dans notre pays, à la fois ponctuelles, foisonnantes et disparates, sans véritable organisation d'ensemble, ce qui n'est pas le cas pour les risques industriels ou de transport (cf. séminaire Gilbert) [3].

Citons :

- les feux de forêts en zone méditerranéenne, exemple actuellement le plus abouti, sur la base d'un fichier d'événements depuis 25 ans (Prométhée) et d'une coopération Agriculture -Intérieur.

- Les rapports de la Mission d'inspection spécialisée de l'environnement (MISE) sur les grands événements de 1'Hexagone de la dernière décennie (le Grand Bornand, Nîmes, Vaison-la-Romaine, Rhône, Var, Hérault...) [4].
- L’exercice 1998 des départements de la zone de défense sud-ouest sur une inondation de grande ampleur du Bassin Adour-Garonne.

- Le fichier "CATNAT" en cours de constitution à la Caisse Centrale de Réassurance, pour tenter une exploitation des données des assureurs sur les événements classés CATNAT par l'Etat.

Face à cette situation "montante" d'initiatives partielles et dispersées, la ministre de l'Aménagement du Territoire et de l'Environnement a demandé à la MISE d'examiner les conditions d'organisation d'un Retour d'expérience sur les risques naturels dans notre pays. Il est rendu compte ici de cette mission, conduite ces derniers mois par MM. Berthelemy, Ingénieur général des Mines, C. Lefrou, Ingénieur général des Ponts et Chaussées, G. Couzy, Ingénieur en chef des Ponts et Chaussées et moi-même. [5].

\section{II $\square$ LES ENJEUX DU RETOUR D'EXPÉRIENCE}

\subsection{L'inondation : un poids socio-économique non négligeable}

Les événements de la dernière décennie ont marqué les esprits, qu'il s'agisse d'inondations rapides, torrentielles alpines, méditerranéennes et/ou urbaines, déjà citées, ou d'inondations de plaine (Oise, Meuse, Vilaine, Rhin, Rhône...).

Le premier type est mortel (une large centaine de victimes en dix ans en France), le second peut coûter très cher (cf infra). 
Jusqu'à une date récente, l'inondation, qui intéresse $10 \%$ de notre territoire et une dizaine de milliers de communes, générait la plus grande partie des indemnisations "CATNAT" (aujourd'hui, la subsidence est la préoccupation majeure...).

L'inondation est à l'origine des événements les plus ordinaires comme les événements parmi les plus graves possibles dans l'Hexagone, avec des dégâts probables considérables (Seine : 50 milliards de francs, Loire : 15/40 milliards...) [6].

La politique de rigueur de l'Etat depuis 1994 vis-à-vis de la gestion des zones inondables pour réduire la vulnérabilité engendre d'ailleurs un dialogue difficile avec les collectivités.

Au-delà des dommages aux biens et aux activités, les impacts psychologiques des inondations même non mortelles sont importants ( $\mathrm{cf}$ le livre de Madeleine CHAPSAL "L'inondation").

Trois objectifs peuvent être assignés au retour d'expérience :

\subsection{Accident banal ou catastrophe?}

Depuis la loi d'indemnisation de 1982, 30000 communes ont été déclarées au moins une fois en état de catastrophe naturelle, certaines plus de 10 fois ! La moyenne des dossiers d'indemnisation est de 5 millions de francs/dossier. Autant dire que notre pays, connu pour la modération de son climat, a peut-être été le théâtre d'une évolution des phénomènes naturels, mais a sûrement vécu une dérive sémantique en prenant l'habitude, du fait des mécanismes d'indemnisation très confortables et peu responsabilisants, de nommer "catastrophe" un banal accident météo. La Commission CATNAT s'efforce actuellement de redonner un sens aux mots, mais il est certain qu'un "retour d'expérience" codifié sur chaque événement permettrait de "hiérarchiser" l'ensemble. C'est un premier objectif et un enjeu national.

\section{- 2.3. Tirer les "enseignements" au niveau local}

Il est certain que c'est aux partenaires de la crise (maire, préfet, société civile) d'évaluer localement la façon dont se sont déroulés les événements et leurs impacts. Le représentant de la Direction de la Défense et de la Sécurité Civile (DDSC) vient de présenter cet aspect des choses pour ce qui concerne la gestion de la crise. A côté de la préoccupation du responsable de la sécurité civile pendant la crise, il y a celle de l'aménageur, du bâtisseur, de l'ingénieur. L'analyse du comportement à la crue des milieux naturels, des équipements, des constructions peut permettre des améliorations, des corrections d'erreurs, des précautions nouvelles, voire des réorientations dans l'aménagement. C'est un second objectif et un enjeu d'abord local, quitte à diffuser et transférer les résultats et les méthodes au niveau national.

\subsection{Participer aux échanges internationaux}

La présentation aujourd'hui d'expériences polonaises et italiennes, les nombreuses rencontres générées par la DIPCN [7], avec des pays plus habitués à ces pratiques de retour d'expérience, la situation dramatique au regard des inondations de pays tels que le Bangladesh, I'Inde ou la Chine, dans des contextes naturels, sociaux, culturels, économiques très différents des nôtres, (concernant par exemple les dimensions des phénomènes, la possibilité de s'installer ailleurs, ou le prix de la vie humaine), l'existence d'une "Ingéniérie" de prévision ou de protection, tout milite pour l'élaboration d'un outil français de "retour d'expérience" qui permette de contribuer efficacement au dialogue international. Tel est le troisième objectif.

Pour chacun de ces trois objectifs, la priorité devra être donnée à tel ou tel des éléments ci-après.

\section{III - CONDITIONS DU RETOUR D'EXPÉRIENCE}

\subsection{Trois conditions}

Trois conditions ou outils paraissent nécessaires au succès d'un retour d'expérience :

- Un climat de confiance d'abord. M. CONDEMINE en a parlé. C'est difficile dès qu'il y a des victimes, et de façon plus générale, compte tenu de l'atmosphère ambiante de pénalisation.

- Une banque de données nationale ensuite, qui permette de garder la mémoire des événements et de les comparer.

- Une animation permanente et "interactive" avec et entre les fournisseurs de données enfin : les événements sont peu nombreux pour un même territoire, ce qui est cause de démotivations. Le "retour" des exploitations nationales vers le terrain qui a fourni les données n'est pas toujours fait, ce qui est une seconde source de démotivation à éviter.

Tableau 1. Propositions d'échelle de gravité.

\begin{tabular}{|c|c|c|c|}
\hline \multicolumn{2}{|c|}{ Niveau } & \multirow{2}{*}{$\begin{array}{c}\text { Dommages humains } \\
\text { aucun blessé }\end{array}$} & \multirow{2}{*}{$\begin{array}{c}\text { Dommages matériels } \\
<0,3 \text { Meuro } \\
<2 \mathrm{MF}\end{array}$} \\
\hline 0 & incident & & \\
\hline 1 & accident & 1 ou plusieurs blessés & $\begin{array}{c}\text { entre } 0,3 \text { et } 3 \text { Meuro } \\
\text { entre } 2 \text { et } 20 \mathrm{MF}\end{array}$ \\
\hline 2 & accident grave & 1 à 9 morts & $\begin{array}{l}\text { entre } 3 \text { et } 30 \text { Meuro } \\
\text { entre } 20 \text { et } 200 \mathrm{MF}\end{array}$ \\
\hline 3 & accident très grave & 10 à 99 morts & $\begin{array}{l}\text { entre } 30 \text { et } 300 \text { Meuro } \\
\text { entre } 200 \mathrm{MF} \text { et } 2 \text { GF }\end{array}$ \\
\hline 4 & catastrophe & 100 à 999 morts & $\begin{array}{c}\text { entre } 300 \text { Meuro et } 3 \text { Geuro } \\
\text { entre } 2 \text { et } 20 \mathrm{GF}\end{array}$ \\
\hline 5 & catastrophe majeure & 1000 morts ou plus & $\begin{array}{l}\text { plus de } 3 \text { Geuro } \\
\text { plus de } 20 \mathrm{GF}\end{array}$ \\
\hline
\end{tabular}




\subsection{Une échelle de gravité (Tableau 1)}

On a souligné l'intérêt de disposer d'un outil pour hiérarchiser les événements les uns par rapport aux autres. En nous inspirant des échelles de gravité utilisées pour les risques technologiques, nous proposons une échelle en cinq niveaux reproduite ici. Elle repose sur une estimation de la vie humaine à $20 \mathrm{MF}$ environ, ordre de grandeur utilisé en Europe. Un événement est classé au niveau correspondant à l'une de ses caractéristiques de dommages (humains et matériels).

En complément, un événement pourrait être caractérisé aussi par un indice de "la situation de danger" qui l'a généré.

Cela existe déjà pour la neige par exemple (échelle européenne en 5 niveaux), ou les feux de forêts. Pour l'inondation, les BRAM et ALARM de la Météo mettent en garde vis-à-vis des situations hydro-météorologiques et les services d'annonce de crues donnent de plus en plus souvent des prévisions de propagation de hauteurs. Une codification en niveaux de ces prévisions aurait-elle un sens ? De même, l'aléa - la crue - une fois survenue peut être caractérisée par sa hauteur, sa vitesse, sa surface, sa durée... Une réflexion sur une codification de ces critères en niveaux ne serait-elle pas utile ?

\section{IV — CONTENU ET ORGANISATION POS- SIBLE DU RETOUR D'EXPÉRIENCE}

\section{- 4.1. Un examen d'ensemble qui demande du temps}

Il conviendrait d'attendre du retour d'expérience, un examen d'ensemble de la situation avant, pendant et après la crise, portant sur :

- l'analyse des phénomènes physiques générateurs,

- l'évaluation des dommages humains et matériels,

- le fonctionnement de la chaîne de surveillance et d'alerte,

- la gestion de la crise et de la post-crise,

- le retour à la normale,

- les préconisations en termes de prévention.

Les intervenants précédents ont surtout mis l'accent sur la crise, la post-crise, l'évaluation des dommages [8]. Je voudrais dire un mot ici de deux aspects :

- l'analyse des phénomènes,

- l'aménagement.

L'expérience de missions récentes ou en cours à la MISE montre que :

- le retour d'expérience peut être l'occasion de "recadrer" les paramètres hydrométéorologiques et hydrauliques d'un bassin, y compris en ordre de grandeur. Cela nécessite expertise et parfois modélisation, pour lesquelles le temps nécessaire relève plus de mois que de semaines, d'autant qu'il peut s'agir de remises en cause de données considérées comme acquises, ce qui nécessite le temps d'un nouveau consensus.

- Le comportement de l'aménagement à l'inondation est riche d'enseignement, sur l'état des ouvrages et des milieux, les éventuelles erreurs d'appréciation sur la localisation des urbanisations, la pertinence des modalités constructives. Un bilan détaillé peut conduire à revoir les documents d'aménagement et d'urbanisme, ce qui suppose là encore du temps pour arriver à un consensus.

\subsection{Une opération en trois étapes}

Ce qui précède conduit à préconiser un retour d'expérience en trois étapes. Citons ici le rapport de la MISE [5] : 1. Une première étape devrait être le recueil dans les jours qui suivent l'accident ou la catastrophe des indications immédiatement disponibles. Il faut souligner que certaines de ces informations sont susceptibles de disparaître rapidement ; par exemple, des photographies aériennes d'une crue doivent être prises dès que les conditions météorologiques le permettent. Dans un délai court, il est également utile de recueillir "à chaud" les réactions des intervenants et de la population, sans gêner les secours. Une première information devrait être rassemblée dans un délai de l'ordre du mois. Cette information sera évidemment incomplète sur de nombreux points et devrait être complétée ultérieurement. 2. Une deuxième étape, dans un délai de l'ordre de 3 à 6 mois, devrait comprendre cinq volets : une information plus complète sur l'accident ou la catastrophe, (caractéristiques du phénomène naturel générateur, sa fréquence de retour, les dommages), une analyse de la situation antérieure en ce qui concerne l'organisation de la prévention, (existence de plans de prévention, prise en compte dans les documents d'urbanisme, existence d'ouvrages de protection), une évaluation de la prévision (surveillance, vigilance, alerte), une description de la gestion de la crise (timing, relations entre acteurs) et enfin les premières décisions prises après l'accident ou la catastrophe (indemnisation, mais aussi décision d'élaboration ou de révision des plans de prévention, des documents d'urbanisme, décisions relatives aux ouvrages de protection). Le délai est nécessaire pour l'aboutissement des premières études locales sur le phénomène et ses conséquences. Dans ce délai, on devait également préciser les informations sur l'indemnisation des victimes et en particulier le montant des indemnisations commune par commune. La Caisse Centrale de Réassurance est en train de modifier son système d'information statistique pour disposer de cette information.

Une attention particulière serait portée à la vulnérabilité des enjeux, en général mal connue.

3. Une troisième étape devrait en général être prévue dans un délai d'environ deux ans. Cette étape devrait principalement porter sur les enseignements tirés de l'accident ou de la catastrophe et en particulier vérifier l'engagement des mesures de prévention (plans de prévention, ouvrages, information, formation) décidées au lendemain de l'accident. Il faut alors s'assurer que l'on a pas trop vite oublié les enseignements de l'accident survenu.

Par ailleurs, pour permettre divers travaux statistiques, pour la banque de données nationale, il est nécessaire que l'on dispose pour chaque événement d'un certain nombre d'informations sous une forme rigoureusement standardisée. Cela ne préjuge évidemment pas de l'intérêt de disposer de documents sous des formes variées.

Une fiche de notification assez courte (4 pages) pourrait être utilisée systématiquement pour tout événement. Sous chaque rubrique, on devrait trouver une description très succincte complétée par des indications codifiées.

Une telle fiche devrait être adressée dans le délai d'un mois par le préfet à l'organisme chargé de la centralisation au niveau national et éventuellement à l'échelon intermédiaire ou thématique (cf infra) ; dans ce délai, la fiche serait généralement incomplète et devrait ensuite être complétée dans un délai de 6 mois maximum. 
Tableau 2. Organisation à trois échelons

\begin{tabular}{|c|c|c|}
\hline & Structure & Tâches \\
\hline $\begin{array}{l}\text { 1. Pôle national } \\
\text { de retour } \\
\text { d'expérience }\end{array}$ & $\begin{array}{l}\text { - type BARPI (jumelé ou non } \\
\text { avec lui, en un Centre de retour } \\
\text { d'expérience...) ou GIP (équipe } \\
\text { de } 5 \text { à } 10 \text { personnes) } \\
\text { - appuyé sur un Conseil } \\
\text { d'orientation de partenaires }\end{array}$ & $\begin{array}{l}\text { - traite les retours "locaux" et anime les réseaux territoriaux } \\
\text { et thématiques (y compris stages de formation), } \\
\text { - gère et établit le réseau de bases de données et fonds } \\
\text { documentaires, } \\
\text { - établit et diffuse des rapports annuels et études thématiques, } \\
\text { - assure la liaison avec la Sécurité Civile (DDSC/INESC) et } \\
\text { les autres organismes ministériels (IFEN) ou autres (CCR...), } \\
\text { - développe les contacts européens et internationaux. }\end{array}$ \\
\hline $\begin{array}{l}\text { 1. Réseau } \\
\text { territorial }\end{array}$ & $\begin{array}{l}\text { - par Département, le Préfet } \\
\text { désigne une équipe de } \\
\text { correspondants ( } 2 \text { à } 4 \text { personnes, } \\
\text { DDSC, DDE, DDAF...) }\end{array}$ & $\begin{array}{l}\text { - établit les "notifications d'événements" (fiche } 4 \text { pages), } \\
\text { - peut constituer le noyau permanent de l'Etat du "groupe } \\
\text { local " constitué par le Préfet ou le Maire en cas de CATNAT, } \\
\text { - participe aux exercices de gestion de crise, } \\
\text { - travaille avec la CARIP, } \\
\text { - regroupe les notifications d'événements départementaux. }\end{array}$ \\
\hline $\begin{array}{l}\text { 2. Echelon } \\
\text { intermédiaire } \\
\text { - administratif }\end{array}$ & $\begin{array}{l}\text { - par Région, Zone ou } \\
\text { Bassin, la Diren ou les Diren } \\
\text { concernées étant les pivots }\end{array}$ & $\begin{array}{l}\text { - effectue toute synthèse et animation des Départements } \\
\text { utiles en accord avec l'échelon national, } \\
\text { - participe aux exercices régionaux, zonaux... }\end{array}$ \\
\hline - thématique & $\begin{array}{l}\text { - Montagne (RTM) } \\
\text { - Littoral (STCPMVN) } \\
\text { - Forêts (DPFM) } \\
\text { - Bassin (DIREN/DE) } \\
\text { - Mouvement de terrain } \\
\text { (BRGM) }\end{array}$ & $\begin{array}{l}\text { - participe aux retours d'expérience spécifiques du milieu, } \\
\text { - gère les bases de données spécialisées du milieu }\end{array}$ \\
\hline
\end{tabular}

Le retour d'expérience ne devrait pas se limiter aux événements à venir ; il faudrait, autant que possible, reprendre les dossiers CATNAT établis depuis 1982 pour les intégrer dans les bases de données. Il faudrait de même reprendre les grandes catastrophes survenues dans le passé et pour lesquelles on dispose d'informations pour étoffer les bases de données. Il ne faut pas se cacher qu'il s'agit là d'un travail de longue haleine, proche parfois de la "recherche historique".

\subsection{Une organisation en trois échelons}

Comme il a été dit, le système à mettre en place doit fonctionner en réseau. Le tableau 2, extrait du rapport cité [5], propose un fonctionnement à trois étages avec :

- un pôle national, constitué d'une équipe de haut niveau, aux profils capables de construire et gérer une base de données, d'animer un réseau de correspondants locaux.

- un réseau territorial, centré sur le département, constitué d'une équipe interadministrative validée par le Préfet, qui intervient auprès de chaque "groupe local" constitué pour un événement donné, et assure la notification des données au niveau central (notamment la fiche citée en 4.2.).

- un échelon intermédiaire administratif (région) ou thématique par milieu (bassin pour les inondations).

\section{$\mathrm{V} \square$ CONCLUSION}

Il faut souhaiter que l'année 2000 voit l'émergence d'une organisation française dans ce domaine. On voit qu'un mouvement s'amorce. Il reste à le coordonner entre Administra- tions, mais aussi avec les Collectivités et les Acteurs de la Société Civile. Des opportunités existent ; en voici une : Yves DAUGE, député Maire de Chinon, est en charge de propositions pour l'organisation éventuelle d'un lieu de débat pour la gestion des inondations. Comment faire vivre un tel lieu sans l'alimenter ? Un retour d'expérience organisé peut et devrait y contribuer.

\section{Références}

[1]. Introduction aux Cyndiniques, sous la direction de JC. WYBO, Edition ESKA, 1998,

[2]. Rapports de l'instance d'évaluation des politiques publiques de prévention des Risques naturels, rapport Bourrelier. Documentation française, 1997.

[3]. Séminaire CNRS, "Approche organisationnelle et croisée du retour d'expérience", sous la direction de Claude Gilbert . CNRS, 1998/99.

[4]. Rapports de la MISE sur des événements, MATE 1997/1999.

[5]. Rapport de la MISE sur l'organisation du Retour d'expérience, MATE, DPPR, 1999.

[6]. Colloque SHF 1997, la Crue de 1920 en Région Parisienne.

[7]. Conférence de Paris de la DIPCN, Le retour d'expérience de l'événement climatique de I'hiver 97/98 au Canada, M. DENIS, Actes du Colloque, $97 / 19$ juin 1999, MATE.

[8]. Appel d'offres "Evaluation et prise en compte du Risque par la Société". Programme de recherche MATE/DGAD, 1999, Propositions B. Ledoux, Consultant. 